\title{
GROUPS ISOMORPHIC TO THEIR NON-NILPOTENT SUBGROUPS
}

\author{
by HOWARD SMITH and JAMES WIEGOLD
}

\author{
(Received 4 November, 1996; revised 26 April, 1997)
}

\begin{abstract}
1. Introduction. We were concerned in [12] with groups $G$ that are isomorphic to all of their non-abelian subgroups. In order to exclude groups with all proper subgroups abelian, which are well understood in the finite case [7] and which include Tarski groups in the infinite case, we restricted attention to the class X of groups $G$ that are isomorphic to their nonabelian subgroups and that contain proper subgroups of this type; such groups are easily seen to be 2-generator, and a complete classification was given in [12, Theorem 2] for the case $G$ soluble. In the insoluble case, $G / Z(G)$ is infinite simple [12; Theorem 1], though not much else was said in [12] about such groups. Here we examine a property which represents a natural generalisation of that discussed above. Let us say that a group $G$ belongs to the class $\mathcal{W}$ if $G$ is isomorphic to each of its non-nilpotent subgroups and not every proper subgroup of $G$ is nilpotent. Firstly, note that finite groups in which all proper subgroups are nilpotent are (again) well understood [9]. In addition, much is known about infinite groups with all proper subgroups nilpotent (see, in particular, [8] and [13] for further discussion) although, even in the locally nilpotent case, there are still some gaps in our understanding of such groups. We content ourselves in the present paper with discussing finitely generated $\mathcal{W}$-groupsnote that a $\mathcal{W}$-group is certainly finitely generated or locally nilpotent. We shall have a little more to say about the locally nilpotent case below.

Again there is a decent classification theorem in the soluble case and something of an observation in the insoluble case. We present our main theorem in a form that contains implicit reference to the previous results from [9] and [12]. Briefly, we have from [12] that a finitely generated soluble X-group is abelian-by-finite and either (a) nilpotent, with a very restricted structure or (b) non-nilpotent and either (i) "approximately" the central factor group of $Z$ wr $C_{p}$ for some prime $p \leq 19$ or (ii) an extension of a finite elementary abelian group by an infinite cyclic group (again with further restrictions). A finite non-nilpotent group with all proper subgroups nilpotent is, in particular, an extension of a $p$-group by a cyclic $q$-group for distinct primes $p$ and $q[11 ; 9.1 .9]$.

Our main results are as follows.
\end{abstract}

THEOREM 1. Let $G$ be a finitely generated soluble group and let $F=$ Fitt $G$. If $G \in \mathcal{W}$ then

(a) $F$ is nilpotent and $G / F$ has prime order $q$, and either

(b) $G$ is isomorphic to each of its non-abelian subgroups or

(c) $G=T\langle x\rangle$ for some non-abelian normal subgroup $T$ and element $x$ of infinite order, and each of the following holds.

(i) $x^{q}$ is central in $G$ and $T$ is a finite p-group, where $p$ and $q$ are distinct primes.

(ii) $G /\left\langle x^{q}\right\rangle$ is a (finite) non-nilpotent group with every proper subgroup nilpotent.

(iii) $\Phi(T)=T^{\prime}$ and $G / T^{\prime}$ is isomorphic to each of its non-abelian subgroups.

(iv) For each $k=1, \ldots, q-1$ there exists an inner automorphism $\alpha$ of $T$ such that $\bar{x}$ is conjugate either to $\alpha \bar{x}^{k}$ or to $\alpha \bar{x}^{-k}$ in Aut $T$, where $\bar{x}$ denotes the image of $x$ under the natural map into Aut $T$.

Further, if $G$ satisfies properties (i) to (iv) of (c) then $G \in \mathcal{W}$.

Glasgow Math. J. 40 (1998) 257-262. 
THEOREM 2. Let $G$ be a finitely generated insoluble $\mathcal{W}$-group and let $F=$ Fitt $G$. The $F$ is nilpotent and $G / F$ is infinite simple.

Part (iv) of (c) above is not very satisfactory as it involves reference to a condition for finite groups that is not easily understood. From the discussion of finite, minimal nonnilpotent groups in [9] and from the fact that condition (iv) imposes a similar restriction on $x$ when viewed as an automorphism of $T / T^{\prime}$, we can deduce several further conditions that our group $G$ must satisfy; e.g. $T / T^{\prime}$ has even rank and $T^{\prime}$ is central in $G$. However, we have left open the problem of obtaining a more useful description of finite groups satisfying (iv). This is a convenient point at which to thank the referee for pointing out that our previous version of (iv) was incorrect.

If $G$ is a locally nilpotent $\mathcal{W}$-group that is also soluble, then it is possible to prove that $G$ is either a Fitting group or of finite rank. In the latter case one may determine the structure of $G$ precisely. If $G$ is torsion free, soluble and locally nilpotent then $G$ is nilpotent, but there do exist "mixed" $\mathcal{W}$-groups of infinite rank that are soluble but not nilpotent. It is envisaged that these results and others will be the subject of a future article by the present authors.

2. Proofs. We begin with a very easy lemma.

LEMMA 1. Let $G$ be a group, $N$ a normal nilpotent subgroup of $G$ such that $G=N\langle x\rangle$ for some element $x$. If $M$ is a $G$-invariant subgroup of $N$ such that $\langle M, x\rangle$ is nilpotent then $M \leq Z_{n}(G)$ for some integer $n$.

Proof. For each $i \geq 0, G$ acts nilpotently on each factor $\left(M \cap Z_{i+1}(N)\right) /\left(M \cap Z_{i}(N)\right)$, where $Z_{i}(N)$ is the $i$ th term of the upper central series of $N$.

Our next result is also easy to prove, but it depends on a deep theorem of Kropholler [4].

Lemma 2. If $G \in \mathcal{W}$ and $G$ is finitely generated soluble then $G$ has finite rank.

Proof. By [4] we need only show that $G$ has no $C_{p}$ wr $C_{\infty}$ sections where $p$ is a prime. Assuming the result false, there is a 2-generator subgroup $H$ of $G$ and a normal subgroup $K$ of $H$ such that $H / K \cong C_{p}$ wr $C_{\infty}$; let $B / K$ be the base group of $H / K$, so that $B / K$ is infinite abelian and of exponent $p$. Write $H=B\langle x\rangle$; since $H / K$ is not nilpotent-by-finite the subgroup $B\left(x^{2}\right)$ is not nilpotent and is therefore 2-generator. However, even $B\left(x^{2}\right) / K$ is not 2-generator, and we have a contradiction.

Lemma 3. Let $G$ be a finitely generated soluble $\mathcal{W}$-group and let $F=$ Fitt $G$. Then $F$ is nilpotent and $G / F$ has prime order.

Proof. Since $F$ is locally nilpotent we have $F \not G$ and hence $F$ is nilpotent; also $G^{\prime}$ is nilpotent and so $F \geq G^{\prime}$, which implies that $F=$ Fitt $H$ for all $H$ satisfying $F \leq H \leq G$. Choosing $g \in G \backslash F$ we now have $F(g)$ normal in $G$ and hence non-nilpotent and hence isomorphic to $G$, so $G / F$ is certainly cyclic, say $G=F\langle x\rangle$. If $G / F$ is finite then there exists $y \in G \backslash F$ such that $y$ has prime order $p \bmod F$; clearly $G=F\langle y\rangle$ in this case. Suppose for a contradiction that $G / F$ is infinite and let $K$ be the hypercentre of $G$. Then $K \leq F$ and we show 
that $F / K$ is infinite. If $F / K$ is finite then $G / K$ is finite-by-cyclic and hence centre-byfinite, giving the contradiction that $G$ is nilpotent-by-finite. Further, if $L$ is any proper $G$-invariant subgroup of $F$ and $F / L$ torsion-free then $\langle x\rangle L$ has smaller Hirsch length than $G$ and is therefore nilpotent, so that $L \leq K$. (Here we have used Lemmas 1 and 2.) It follows that $F / K$ is torsion free; since $F / F^{\prime}$ is certainly infinite we have $F / K$ abelian.

We now show that $G / K \in \mathcal{W}:$ since $G / K$ is non-nilpotent, it suffices to prove that $G / K$ is isomorphic to each of its non-nilpotent subgroups. Let $H / K$ be such a subgroup; then $H \cong G$ and the hypercentre $K^{*}$ of $H$ contains $K$. But $G / K \cong H / K^{*}$, and consideration of Hirsch length gives $K=K^{*}$ and hence $G / K \cong H / K$ as required. Since $G$ is not nilpotent-by-finite, neither is $G / K$, so Fitt $(G / K)=F / K$ and we may factor by $K$ and thus assume that $G$ has trivial centre and $F$ is abelian.

Suppose next that $G=F M$ for some nilpotent subgroup $M$ of class $r$, say. Then $M \cap F \triangleleft G$ and $[M \cap F, r G]=[M \cap F, r M]=1$, so that by Lemma $1 M \cap F \leq K=1$, and we have $M \cong M / M \cap F \cong G / F$, which is cyclic. Thus if $H$ is an arbitrary nilpotent subgroup of $G$ then either $H \leq F$ and so $H$ is abelian, or $H F$ is not nilpotent and hence $H F \cong G$ and $H$ is cyclic. It follows that every non-abelian subgroup is isomorphic to $G$ and hence that $G$ is abelian-by-finite, by [12, Theorem 2]. This contradiction completes the proof of the lemma.

Much of the proof of Theorem 1 is dealt with by our next result.

Lemma 4. Let $G$ be a finitely generated soluble $\mathcal{W}$-group and suppose that $G=T\langle x\rangle$ for some periodic normal subgroup $T$ and element $x$. Then $T$ is a finite $\mathrm{p}$-group for some prime $p$, and $G$ is as described in part (c) of Theorem 1.

Proof. Clearly $T \cap\langle x\rangle=1$ since $G$ is not periodic; also, $G / T^{\prime}$ is finitely generated and metabelian and therefore satisfies $\max -n$ [2], so that $T / T^{\prime}$ is finite (using Lemma 2) and hence $T$ is finite (since it is nilpotent). If $T=U \times V$ for some non-trivial $G$-invariant subgroups $U$ and $V$ then each of $U\langle x\rangle$ and $V\langle x\rangle$ is nilpotent and hence $T \leq Z_{n}(G)$ for some $n$, by Lemma 1 . By this contradiction, $T$ is a $p$-group for some prime $p$. Write $F=$ Fitt $G$, so that $G / F$ has prime order $q$, say, by Lemma 3 and we have $F=T\left\langle x^{q}\right\rangle$, which is nilpotent. Thus $x^{q p^{k}}$ centralises $T$ for some $k \geqslant 0$. If $T\left\langle x^{p^{k}}\right\rangle$ were nilpotent then, since $p \neq q$, we would have $G=T\langle x\rangle$ nilpotent, a contradiction. Replacing $x$ by $x^{p^{k}}$ if necessary, we have $\left[T, x^{q}\right]=1$. Hence part (c)(i) of the theorem is established. Now suppose that $H$ is a non-nilpotent subgroup of $G$ with $x^{q} \in H$. Since $H \cong G$ we have $T \leq H$ and hence $F \leq H$; this gives $H=G$, and (ii) follows.

Next, we have from [9; Satz 3] that $T / T^{\prime}$ has exponent $p$ and hence $\Phi(T)=T^{\prime}$. Since every proper $\langle x\rangle$-invariant subgroup of $T$ is centralised by $x$ it follows easily from Maschke's Theorem that $T / T^{\prime}$ is a chief factor of $G$. Let $K / T^{\prime}$ be a non-nilpotent subgroup of $G / T^{\prime}$; since $T^{\prime}$ is invariant under any isomorphism $G \rightarrow K$ we see that $G / T^{\prime} \in \mathcal{W}$. (Note that $G / T^{\prime}$ is not nilpotent, by Lemma 1.) We claim that every nilpotent subgroup $H / T^{\prime}$ of $G / T^{\prime}$ is abelian. Clearly we may assume for this purpose that $T^{\prime}=1$ and $Z(G) \leq H$, so that $H T=F$ or $H T=G$ and hence either $H \leq F$, which is now abelian, or $H \cap T \triangleleft G$ and hence $H \cap T=1$ (since $T \leq H$ ). In the latter case we have $H$ cyclic, and so the claim is established. Part (iii) now follows.

Finally, let $k$ be an integer with $1 \leq k \leq q-1$, and write $H=T\left\langle x^{k}\right\rangle$. There is an isomorphism $\theta: G \rightarrow H$ and clearly $\theta$ restricts to an automorphism of $T$. Further, since $x$ has infinite order we have $x \theta=a x^{ \pm k}$ for some $a \in T$. Let $\alpha$ denote the inner automorphism of 
$T$ induced by conjugation by $a$ and let $u \in T$. Then we have $u \bar{x} \theta=u \theta \alpha \bar{x}^{ \pm k}$, and hence it follows that $\theta^{-1} \bar{x} \theta=\alpha \bar{x}^{ \pm k}$, thus establishing (iv) and completing the proof of Lemma 4 .

LEMma 5. Let $X$ be a finitely generated, torsion-free nilpotent group of class (exactly) c and suppose that, for some element y of $X$ and some infinite set $\pi$ of primes, $X \cong(y) Z_{c-1}(X) X^{p}$ for all $p \in \pi$. Then $X$ is abelian.

Proof. Suppose the result false, and let $X$ be a counterexample with $c$ minimal, so that $c \geq 2$. Write $X_{p}=\langle y\rangle Z_{c-1}(X) X^{p}$ for each $p$; then $Z\left(X_{p}\right)=Z(X)=Z$, say, for if $g \in Z\left(X_{p}\right)$ then $\left[g, x^{p}\right]=1$ for all $x \in X$, which implies that $[g, x]=1$ for all $x$ since centralisers are isolated [3; Corollary 10 of Lemma 4.7]. It follows that $Z$ is invariant under any isomorphism $X \rightarrow X_{p}$, and thus $X / Z \cong X_{p} / Z=(\langle y\rangle Z / Z)\left(Z_{c-2}(X / Z)\right)(X / Z)^{p}$. By minimality we have $X / Z$ abelian and hence $c=2$. For $p \in \pi$ we have $Z / X^{\prime} \cong Z / X_{p}^{\prime}$; but $p$ divides $\left|X^{\prime} / X_{p}^{\prime}\right|$ and hence divides the order of the torsion subgroup of $Z / X^{\prime}$. However this cannot hold for infinitely many $p$, and we have the required contradiction.

Proof of Theorem 1. Let $F=$ Fitt $G, T$ the torsion subgroup of $F$. By Lemma 3, $F$ is nilpotent and $G=F\langle x\rangle$ for some $x$ of prime order $q \bmod F$. If $T\langle x\rangle \cong G$ then Lemma 4 applies, so suppose that $T\langle x\rangle$ is nilpotent. Then $T \leq Z_{n}(G)$ for some $n$, by Lemma 1 . If $H$ is an arbitrary non-nilpotent subgroup of $G$ containing $T$ then Fitt $H$ equals $F \cap H$, since $|H / F \cap H|$ divides $q$. It follows that $T$ is the torsion subgroup of Fitt $H$ and hence invariant under any isomorphism from $G$ to $H$; since $G / T$ is not nilpotent we thus have $G / T \in \mathcal{W}$.

Assume first that $T=1$, so that $F$ is torsion free and nilpotent of class $c$, say. If $X_{p}=:\langle x\rangle F^{p} Z_{c-1}(F)$ is nilpotent for some prime $p$ then we may apply Lemma 1 to deduce that $G / Z_{r}(G)$ is finite for some integer $r$, and then $\gamma_{r+1}(G)$ is finite [10; Corollary 2 of Theorem 4.21] and hence trivial (since $T=1$ ), a contradiction. Writing $F_{p}=$ Fitt $X_{p}$, we thus have $\left|X_{p}: F_{p}\right|=q$ and hence $F_{p}=\left\langle x^{q}\right\rangle F^{p} Z_{c-1}(F)$ for each prime $p$. Since $F_{p} \cong F$ for all $p$ we deduce from Lemma 5 that $F$ is abelian.

In the general case, we thus have $F^{\prime} \leq T$, and so $G / T^{\prime}$ is finitely generated and metabelian-by-finite. As in the proof of Lemma 4, $T$ is therefore finite, which gives $F^{\prime}$ finite and hence $F$ centre-by-finite. Let $Z$ be the centre of $F$. If $\langle x\rangle Z$ is nilpotent then, as above, $G / Z_{r}(G)$ is finite for some $r$ and $\gamma_{r+1}(G) \leq T \leq Z_{n}(G)$ for some $n$, a contradiction. Thus $\langle x\rangle Z \cong G$ and it follows that $F$ is abelian. Now $F^{k}$ is torsion free for some positive integer $k$, and the same argument gives $\langle x\rangle F^{k}$ non-nilpotent, so that $G / A$ is finite and cyclic for some torsion-free abelian normal subgroup $A$. We may assume that $G=A\langle x\rangle$. Since $A$ is finitely generated $\exists k \geq 0$ such that $B=:[A, k\langle x\rangle]$ has the same rank as $[A, k+1\langle x\rangle]$ (in fact, $k=0$ if $x$ has finite order). Since $B \neq 1$ we have $B\langle x\rangle$ isomorphic to $G$ and we may assume that $G=B\langle x\rangle$. The map $b \rightarrow[b, x]$ for all $b \in B$ is an epimorphism whose kernel is finite and hence trivial; hence $Z(G) \cap B=1, Z(G)=\left\langle x^{q}\right\rangle$ and $\left.G=B\right]\langle x\rangle$. Let $H$ be a nilpotent subgroup of $G$ that contains $x^{q}$. If $H \leq F$ then $H$ is abelian; otherwise $G=H B$ and so $H \cap B \triangleleft G$ and $\left[H \cap B,{ }_{d} G\right]=[H \cap B, d H]=1$ where $d$ is the class of $H$. It follows that $H \cap B=1$, else we have the contradiction that $Z(G) \cap B \neq 1$. This shows that every nilpotent subgroup of $G$ is abelian and hence that $G$ is isomorphic to each of it non-abelian subgroups.

It remains only to establish the converse. Suppose that $G$ has the properties (i)-(iv) of (c) and let $H$ be a non-nilpotent subgroup of $G$. Since $T^{\prime}$ is in the hypercentre of $G$ and $G / T$ is 
cyclic we have $H \cap T \notin T^{\prime}$; hence $\exists t \in(H \cap T) \backslash T^{\prime}$. By (i), $H\left\langle x^{q}\right\rangle /\left\langle x^{q}\right\rangle$ is non-nilpotent and so $H\left\langle x^{q}\right\rangle=G$, by (ii) and it follows that $\left\langle(\rangle^{(5)} \leq H\right.$. Now $G / T^{\prime}\left\langle x^{q}\right\rangle$ has every proper subgroup abelian, by (i) and (ii), and so $T / T^{\prime}$ is a chief factor of $G$ (again using Maschke's Theorem). If $\langle t\rangle^{(G)}<T$ then we have $\langle t\rangle T^{\prime}$ centralised by $x$ and hence normal in $G$, giving the contradiction $t \in T^{\prime}$. Thus $\langle t\rangle^{\langle G\rangle}=T$ and so $T \leq H$. Let $k$ be the least positive integer such that $x^{k} \in H$; then $q$ does not divide $k$ since $H \not F$, and $H=T\left\langle x^{k}\right\rangle$. With the notation as in (iv), suppose that $\alpha$ represents conjugation by the element $a$ of $T$ and that $\theta^{-1} \bar{x} \theta=\alpha \bar{x}^{ \pm k}$, where $\theta \in$ Aut $T$. The assignment $u \rightarrow u \theta$ for all $u \in T, x \rightarrow a x^{ \pm k}$ is easily shown to determine an isomorphism from $G$ to $H$, and the proof of Theorem 1 is complete.

We remark that, with the above notation, $G=\langle t, x\rangle$ for any $t \in T \backslash T^{\prime}$; in particular, of course, $G$ is 2-generator. This fact could have been established at the outset, since a finitely generated soluble group with every 2-generator subgroup nilpotent is itself nilpotent [1]. Note also that an easy consequence of our classification theorem is that, for a given finitely generated soluble $\mathcal{W}$-group $G$, there is an upper bound for the class of the nilpotent subgroups of $G$.

Proof of Theorem 2. With the notation as given, $F$ is clearly nilpotent. Also, if $F<N \leq G$ and $N \triangleleft G$, then $N$ is non-nilpotent and hence isomorphic to $G$; also $F=\operatorname{Fitt}(N)$ and so $N / F \cong G / F$. Suppose that $G / F$ is not simple and choose a proper such subgroup $N$ and $g \in G \backslash N$; then $N\langle g\rangle \cong G$ and so $G$ has a non-trivial finite image. Every finitely generated non-trivial nilpotent subgroup certainly has a finite image and so $G$ is locally graded. By [6], $G / F$ is locally graded, so that $G / F$ has a non-trivial finite image and is isomorphic to all of its non-trivial normal subgroups. By [5], $G / F$ is infinite cyclic and we have a contradiction, so $G / F$ is simple. Finally, if $G / F$ is finite then it has a non-nilpotent maximal subgroup $M / F$, by [11; 9.1.9]; then $M \cong G$ and hence $M /$ Fitt $M \cong G / F$. Since Fitt $M \geq F$ we have another contradiction, and the theorem is proved.

\section{REFERENCES}

1. K. W. Gruenberg, Two theorems on Engel groups, Proc. Camb. Phil. Soc. 49 (1953), 377-380.

2. P. Hall, Finiteness conditions for soluble groups, Proc. London Math. Soc. 4 (1954), 419-436.

3. P. Hall, The Edmonton notes on nilpotent groups, QMC notes, London 1969.

4. P. Kropholler, On finitely generated soluble groups with no large wreath product sections, Proc. London Math. Soc. 49 (1984), 155-169.

5. J. C. Lennox, H. Smith and J. Wiegold, A problem on normal subgroups, J. Pure and Applied Alg. 88 (1993), 169-171.

6. P. Longobardi, M. Maj and H. Smith, A note on locally graded groups, Rend. Sem. Mat. Univ. Padova 94 (1995), 275-277.

7. G. A. Miller and H. C. Moreno, Non-abelian groups in which every subgroup is abelian, Trans. Amer. Math. Soc. 4 (1903), 389-404.

8. M. F. Newman and J. Wiegold, Groups with many nilpotent subgroups, Archiv der Math. 15 (1964), 241-250. 130-138.

9. L. Rédei, Die endlichen einstufig nichtnilpotenten Gruppen, Publ. Math. Debrecen 4 (1956),

10. D. J. S. Robinson, Finiteness conditions and generalized soluble groups (2 vols.), (Springer, Berlin-Heidelberg-NewYork, 1972).

11. D. J. S. Robinson, $A$ course in the theory of groups (Springer, Berling-Heidelberg-New York, 1982). 
12. H. Smith and J. Wiegold, Groups which are isomorphic to their nonabelian subgroups, Rend. Sem. Mat. Univ. Padova 97 (1997), 7-16.

13. H. Smith, Groups with few non-nilpotent subgroups, Glasgow Math. J. 39 (1997), 141-151.

Howard Smith

Department of Mathematics

BUCKNELLL UNIVERSITY

LEWISBURG PA 17837

USA
James Wiegold

SCHOOL OF Mathematics

UNIVERSITY OF WALES, CARDIFF

SENGHENNYDD ROAD

CARDIFF CF2 4 YH 